\title{
Moment Distribution Method to Compute the Moment of Continuous Beam Using WPS
}

\author{
Ming Bo Zhao \\ (School of Civil Engineering and Architecture, Southwest University of Science and Technology, \\ MianYang 621010,China) \\ zmwave@126.com
}

Keywords: WPS, moment distribution method, continuous beam, bending moment of beam end Abstract: This paper presents the moment distribution method to compute the moment of continuous beam end, in which the operating functions of WPS are called. In this method, the bending moment of beam end can be calculated in PC system, just inputting the transfer coefficient, the distribution coefficient and constant of loading. This paper can give certainly reference for the construction of examination bank and engineering practice.

\section{Introduction}

One can focus on innovation as WPS helps to compute.

In the past time, the matrix displacement method was popular to analyzed the bending moment of continuous beam $^{[1]}$, and the specialized programming skills and the knowledge of matrix displacement method are required to understand and use that method, so it is not so convenient for the beginners. In this manuscript, the bending moment of the continuous beam can be calculated via WPS software, which has big function of directly input and output interface and built-in spreadsheet functions.

The moment distribution method includes distributing and transferring. The method is easy to understand, but it has tedious computing and error-prone. So it is feasible and convenient with the help of WPS' compute functions to finish the distribution and transmission of moment, improving the efficiency and reducing the errors.

\section{WPS Table Design}

The WPS table design is introduced for the continuous beams with three sections, as shown in Figure 1. The table, as shown in Figure 2, is designed in the Sheet1 of WPS, in which the transfer coefficient, distribution coefficient and constant of loading are stored in rows 3-5 successively(row by row). The distribution and transfer bending moment is put in row rows 6-14, and row 15 is the bending moment of beam end.

\section{Moment distribution and transfer on Node $B$}

To speed up the convergence rate, the maximum absolute value Node is usually the beginning computation node. So node B is chosen in this model.

\section{Moment distribution and transfer on Node B}

According to the computing rules of the moment distribution method, the unbalance moment of Node B can be got by adding the $\mathrm{H} 5$ and $\mathrm{J} 5$ cells and the distribution moment of cell $\mathrm{H} 6$ is the product of the minus of unbalance moment Node B by the distribution coefficient of cell H4. So just input ' $=-(\mathrm{H} 5+\mathrm{J} 5) * \$ \mathrm{H} \$ 4$ ' to cell H6 and '=-(H5+J5)-H6' to cell J6.

Transfer: the transfer moment for cell E6 equals the result of H6 cell multiplies the transfer coefficient E3, so input ' $=\mathrm{H} 6 * \$ E \$ 3$ ' to cell E6. In the same time, the transfer moment for cell M6 equals the result of J6 cell multiplies the transfer coefficient $\mathrm{J} 3$, so input ' $=\mathrm{J} 6 * \$ \mathrm{~J} \$ 3$ ' to cell $\mathrm{J} 6$. 


\section{Moment distribution and transfer on Node $\mathrm{C}$}

The distribution moment of cell M7 can be got by the minus of unbalance moment of Node C 'M5+M6+O5' multiplies the distribution coefficient of cell M4. So just input '=-(M5+M6+O5)*\$M $\$ 4$ ' to the cell $\mathrm{M} 7$ and '=-(M5+M6+O5)-M7' to cell O7.

Transfer: the transfer moment of cell $\mathrm{R} 7$ is ' $=\mathrm{O} 7 * \$ O \mathrm{O} \$ 3$ ', and $\mathrm{J} 7$ is ' $=\mathrm{M} 7 * \$ \mathrm{~J} \$ 33^{\prime}$.

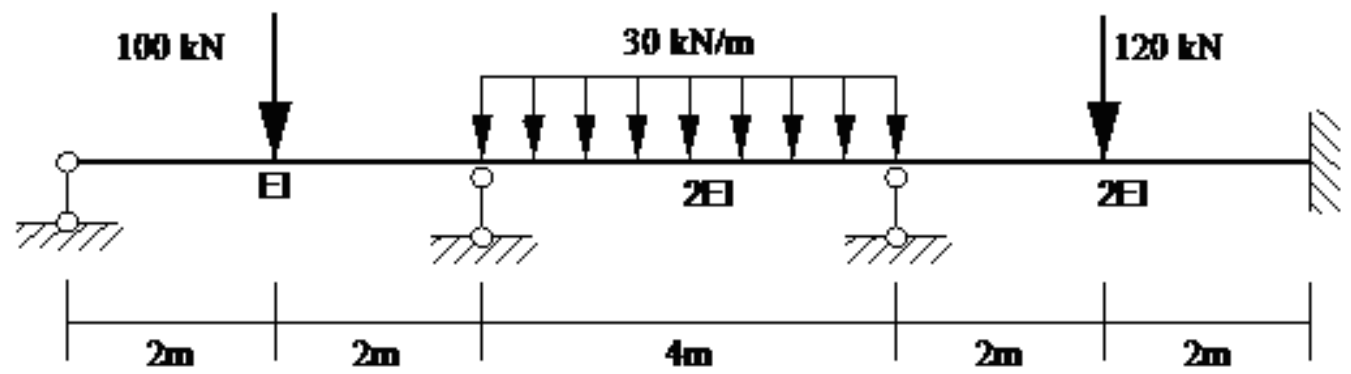

Fig.1 Continuous beam for three sections

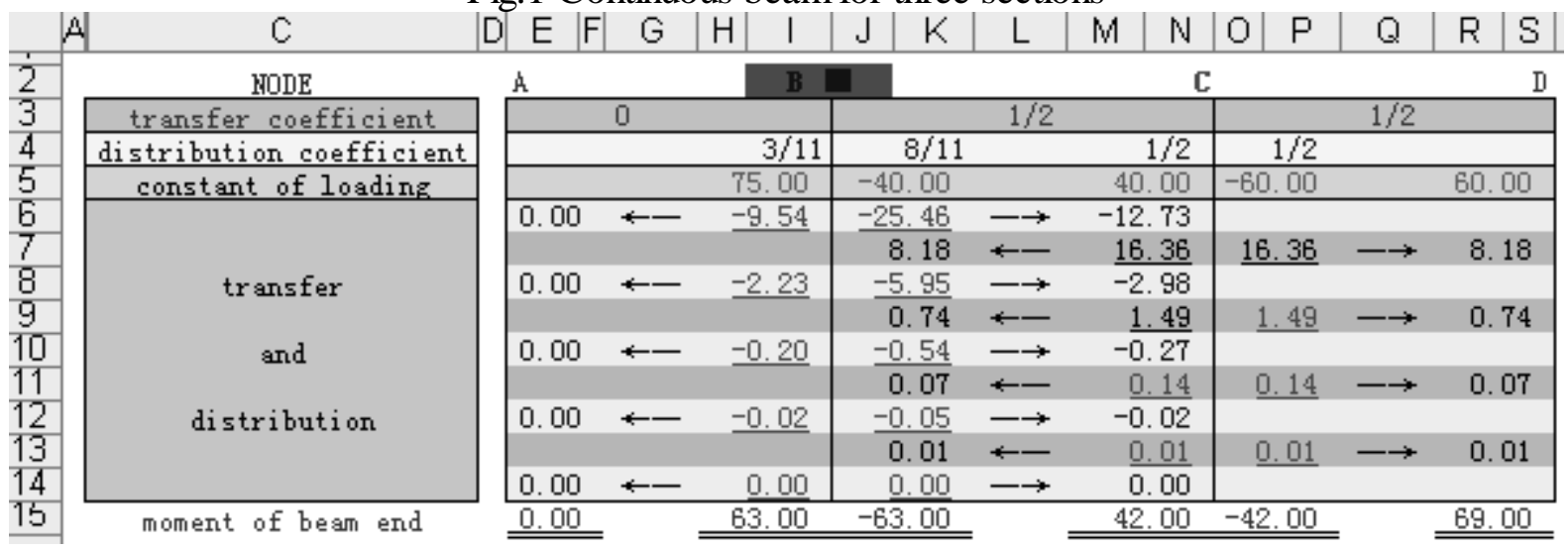

Fig. 2 Moment distribution and transfer on Node B

\section{Redistribution and transfer}

For Node B: Input ' $=-\mathrm{J} 7 * \$ \mathrm{H} \$ 4$ ' to cell H8 and ' $=-\mathrm{J} 7-\mathrm{H} 8$ ' to cell J8. Copy the formula of cell E6 to the cells E8,E10,E12,E14. Similarly, copy the formula of cell M6 to the cells M8,M10, M12, M14.

For Node C:Input ' $=-\mathrm{M} 8 * \$ \mathrm{M} \$ 4$ ' to cell M9 and ' $=-\mathrm{M} 8-\mathrm{M} 9$ ' to cell O9. Copy the formula of cell R7 to the cells R9,R11,R13. Similarly, copy the content of cell J7 to the cells J9, J11, J13.

Further, copy the formula of cell $\mathrm{J} 8$ to the cells J10,J12,J14, copy the formula of cell H8 to the cells $\mathrm{H} 10, \mathrm{H} 12, \mathrm{H} 14$, copy the formula of cell M9 to the cells M11,M13 and copy the formula of cell O9 to the cells $\mathrm{O} 11, \mathrm{O} 13$.

\section{Computing the bending moment of beam end}

The bending moment of beam end is the sum of the distribution bending moment and the transfer bending moment, so input '=SUM(E5:F14)' to the cell E15, then copy the formula of cell E15 to the cells H15,J15,M15,O15,R15 to finish the computation of the bending moment of beam end.

Tagged '__' and '_.' under the distribution bending moment and beam end moment respectively via the built-in functions of WPS sheet. The transfer direction ' $\leftarrow$ ' or ' $\longrightarrow$ ' is shown on the list of transfer coefficient, in the same time two digits after decimal point in the data are displayed by controlling the display mode, as the shown in Figure 2.

\section{Moment distribution and transfer on Node $\mathbf{C}$}

If the node $\mathrm{C}$ is distributing, the corresponding calculation method is similar to the above (Node $\mathrm{B}$ ), the result of computing is shown in Figure 3. 

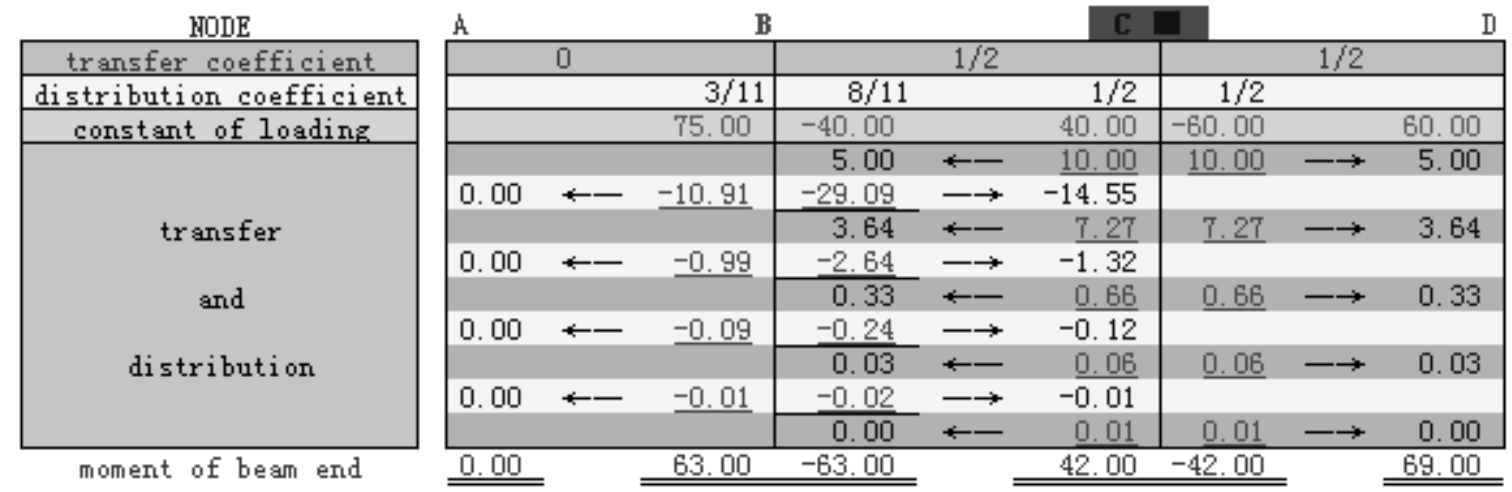

Fig.3 Moment distribution and transfer on Node $\mathrm{C}$

Notes: The distribution coefficient, transmission coefficient should use absolute index, and the relative index for the rest.

According to the test of the author, the error of results will be very small after 9 times distribution and transfer for the most of continuous beam.

\section{Bending moment diagram}

According to the results of computing, the bending moment of the continuous beam end, as shown Figure 1, can be displayed as Figure 4.

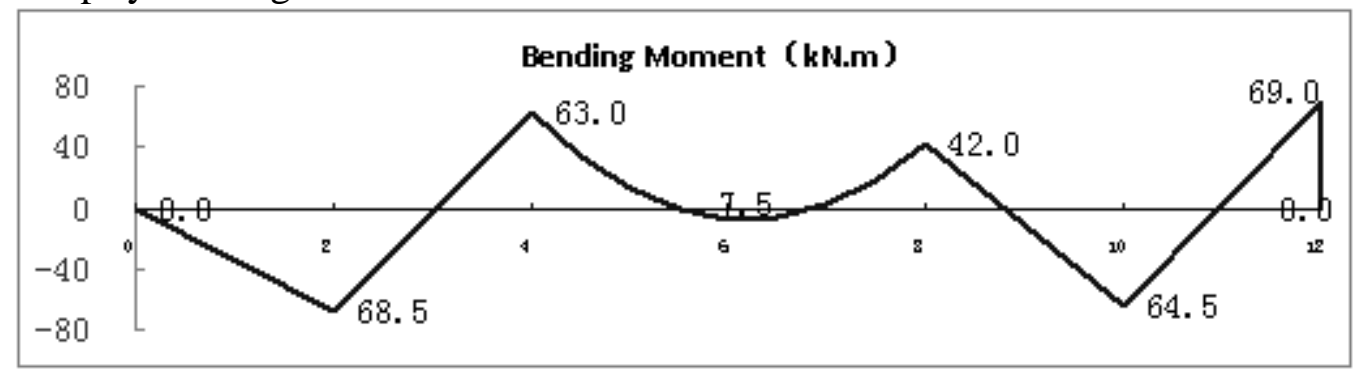

Fig.4 Diagram for bending moment of the continuous beam as shown in Fig.1

\section{Conclusions}

The use of WPS in the table of computing functions and distribution method to calculate the continuous beam bending moment has its unique advantages. Not writing any code needed, just enter the appropriate initial data, one can complete the distribution and transfer of moment fast and accurately. This manuscript can give certainly reference for the construction of examination bank and engineering practice.

\section{References}

[1] Ming Bo Zhao, The study of calculating initial force and deformation of plane truss in EXCEL [J]. Building Science Research of SiChuan, 6(2008)49-52 (in Chinese).

[2] Ming Bo Zhao,Influence Line Analysis of the Plane Trusses Based on EXCEL,Progress in Structures[J],166-169(2012)927-930.

[3] Ming Bo Zhao,Study of force of plane statically determinate plane through VBA in EXCEL [J]. Shanxi Architecture, 19(2005)307-308 (in Chinese). 